\title{
LINEAR ASYMPTOTIC BEHAVIOUR OF SECOND ORDER ORDINARY DIFFERENTIAL EQUATIONS
}

\author{
MATS EHRNSTRÖM \\ Centre for Mathematical Sciences, Lund University, PO Box 118, 22100 Lund, Sweden. \\ e-mail: mats.ehrnstrom@math.lu.se
}

(Received 17 March, 2006; revised 19 October, 2006; accepted 10 November, 2006)

\begin{abstract}
We study the semilinear differential equation $u^{\prime \prime}+F\left(t, u, u^{\prime}\right)=0$ on a half-line. Under different growth conditions on the function $F$, equations with globally defined solutions asymptotic to lines are characterized. Both fixed initial data and fixed asymptote are considered.
\end{abstract}

2000 Mathematics Subject Classification. 34A12, 34E10.

1. Introduction. Let $\mathrm{I} \equiv\left[t_{0}, \infty\right), t_{0} \geq 0$, be a positive half-axis. We consider the semilinear second order differential equation

$$
u^{\prime \prime}(t)+F\left(t, u(t), u^{\prime}(t)\right)=0, \quad t \in \mathrm{I},
$$

where $F: I \times \mathbb{R} \times \mathbb{R} \rightarrow \mathbb{R}$ is continuous in all three variables, and we are seeking solutions $u$ that are twice continuously differentiable on I. In particular, we are looking for conditions that guarantee solutions $u(t)$ of linear asymptotic form, for which there exist constants $c, m \in \mathbb{R}$ such that

$$
|u(t)-c t-m|+\left|u^{\prime}(t)-c\right| \rightarrow 0 \quad \text { as } \quad t \rightarrow \infty .
$$

Ever since [1], much work has been devoted to the investigation of non-oscillatory solutions, i.e. solutions that eventually remain positive (or negative). Especially, one has been interested in the investigation of solutions $u(t)$ in the classes

$$
\{u \text { satisfies }(1.2)\} \subset\{u(t)=c t+m+o(1)\} \subset\{u(t)=c t+o(t)\},
$$

as $t \rightarrow \infty$. The case $c=0$ has been studied separately. The papers $[10,11,17]$ all focus on different classes of solutions, determined by whether $\lim _{t \rightarrow \infty} u(t) / t$ is positive, negative, vanishing or non-existent. Recent papers dealing with solutions $u(t)=c t+$ $o(t)$ are e.g. $[4,13,19]$. In comparison, not as much work has aimed at investigating solutions $u(t)=c t+m+o(1)$. Lately, however, there has been an increasing interest in this, witnessed by $[\mathbf{1 2}, \mathbf{1 4}, \mathbf{1 5}, \mathbf{1 6}]$. Even though the focus of this paper are solutions satisfying (1.2), the results were inspired by work on solutions $u(t)=m+o(1)$ in [6], which in turn had predecessors in $[\mathbf{5}, \mathbf{1 8}]$. (For a different approach, see also [8].) We remark that all solutions dealt with in this paper will be globally defined on I.

Typically, the conditions given in the literature for any of the solutions mentioned above include growth estimates on the function $F\left(t, u, u^{\prime}\right)$, especially concerning the time variable. In this paper, most results are based upon a Lipschitz-like criterion presented as Condition 3.1, which in fact guarantees that all solutions of (1.1) are 
globally defined in time (see [3]). However, Theorem 4.2 and Corollary 4.3 loosen this condition to include equations with solutions that blow-up in finite time. Even though this possibility has been considered, e.g. already in [11], our results are concerned with a smaller class than $u(t)=c t+o(t)$, namely (1.2). Furthermore, Theorem 5.6 is stated directly in terms of the function $F\left(t, u, u^{\prime}\right)$.

Section 2 contains preparations for the proofs to come, defining and investigating some metric spaces. In Section 3 we state necessary and sufficient conditions for all solutions to be of form $u(t)=c t+o(t)$. Sections 4 and 5 then investigate conditions under which solutions with fixed initial data satisfy (1.2) for some $c, m \in \mathbb{R}$, respectively when, for given $c, m \in \mathbb{R}$, there is a solution fulfilling (1.2). Comparisons are made to previous results. All main proofs are based on either the Banach, or the Schauder, fixed point theorem. The Bielecki scaling technique [2] and proper choices of metric spaces (cf. Section 2) are essential.

2. Preliminaries. In this section we will introduce some metric spaces that will be used in the following sections.

By $C^{1}(\mathrm{I})$ we mean the linear space of continuously differentiable real-valued functions $u: \mathrm{I} \rightarrow \mathbb{R}$. Similarly, $C_{b}^{1}(\mathrm{I})$ denotes the subspace of $C^{1}(\mathrm{I})$ of bounded functions with bounded derivative, complete in the metric induced by the extended supremum norm

$$
\|u\|_{1} \equiv \sup _{t \in \mathrm{I}}|u(t)|+\sup _{t \in \mathrm{I}}\left|u^{\prime}(t)\right| .
$$

Throughout the paper, we shall use different norms $\|\cdot\|$ to represent the corresponding metrics $d(u, v)=\|u-v\|$. It is important to note that some function spaces are complete as metric spaces in the metric induced by the norm, even though the members of the space may not be norm bounded! What matters is that their difference is bounded in the corresponding norm. E.g. in the metric given by (2.1), for any $c, m \in \mathbb{R}$ the set $C_{c, m}^{1}(\mathrm{I}) \subset C^{1}(\mathrm{I})$ of functions satisfying (1.2) is closed in $C^{1}(\mathrm{I})$. This makes $C_{c, m}^{1}(\mathrm{I})$ into a complete metric space (even though it is neither linear nor normed in the sense of $\left.\|\cdot\|_{1}\right)$.

We shall consider also the subspace of $C^{1}(\mathrm{I})$ consisting of functions with linear growth, i.e.

$$
\mathcal{X} \equiv\left\{u \in C^{1}(\mathrm{I}): \sup _{t \in \mathrm{I}}\left|\frac{u(t)}{t+1}\right|+\sup _{t \in \mathrm{I}}\left|u^{\prime}(t)\right|<\infty\right\},
$$

endowed with the norm

$$
\|u\|_{\mathcal{X}} \equiv \sup _{t \in \mathrm{I}}\left|\frac{u(t)}{t+1}\right|+\sup _{t \in \mathrm{I}}\left|u^{\prime}(t)\right| .
$$

The norm induces a corresponding metric on $\mathcal{X}$ given by $d(u, v)=\|u-v\|_{\mathcal{X}}$. It can easily be seen that $\mathcal{X}$ is complete with respect to the metric given by $\|\cdot\|_{\mathcal{X}}$. For brevity, we shall write $\mathcal{X}$, referring to the metric space $\left(\mathcal{X},\|\cdot\|_{\mathcal{X}}\right)$. The equivalent norm inducing an equivalent metric -

$$
\|u\|_{\mathcal{X}}^{\max } \equiv \max \left\{\sup _{t \in \mathrm{I}}\left|\frac{u(t)}{t+1}\right|, \sup _{t \in \mathrm{I}}\left|u^{\prime}(t)\right|\right\}
$$


helps us introduce the class of metric subspaces $\mathcal{X}_{c} \subset \mathcal{X}, c \geq 0$, defined by

$$
\mathcal{X}_{c} \equiv\left\{u \in \mathcal{X}:\|u\|_{\mathcal{X}}^{\max } \leq c\right\} .
$$

It is immediate that $\mathcal{X}_{c}$ is closed in $\mathcal{X}$. As a consequence, $\left(\mathcal{X}_{c},\|\cdot\|_{\mathcal{X}}\right)$ is a complete metric space.

REMARK 2.1. Note that for any function $\varphi: \mathrm{I} \rightarrow[m, M], 0<m<M<\infty$, the corresponding norms and induced metrics given by

$$
\|u\|_{1}^{\varphi} \equiv \sup _{t \in \mathrm{I}}\left|\frac{u(t)}{\varphi(t)}\right|+\sup _{t \in \mathrm{I}}\left|\frac{u^{\prime}(t)}{\varphi(t)}\right|,
$$

and

$$
\|u\|_{\mathcal{X}}^{\varphi} \equiv \sup _{t \in \mathrm{I}}\left|\frac{u(t)}{(t+1) \varphi(t)}\right|+\sup _{t \in \mathrm{I}}\left|\frac{u^{\prime}(t)}{\varphi(t)}\right|,
$$

make $\left(C_{c, m}^{1}(\mathrm{I}),\|\cdot\|_{1}\right)$ and $\left(C_{c, m}^{1}(\mathrm{I}),\|\cdot\|_{1}^{\varphi}\right)$, respectively $\left(\mathcal{X},\|\cdot\|_{\mathcal{X}}\right)$ and $\left(\mathcal{X},\|\cdot\|_{\mathcal{X}}^{\varphi}\right)$, equivalent as metric spaces.

3. Linear asymptotic solutions $\boldsymbol{o}(\boldsymbol{t})$. In this section we study necessary and sufficient conditions for the existence of solutions $u(t)$ to (1.1), which satisfy $\left|u^{\prime}(t)-c\right| \rightarrow 0$ and

$$
u(t)=c t+o(t), \quad t \rightarrow \infty .
$$

Throughout the section we shall assume that the following holds true:

Condition 3.1. Suppose that there exists a continuous function $k: \mathrm{I} \rightarrow[0, \infty)$, such that for any $u, u^{\prime}, v, v^{\prime} \in \mathbb{R}, t \in \mathrm{I}$,

$$
\left|F\left(t, u, u^{\prime}\right)-F\left(t, v, v^{\prime}\right)\right| \leq k(t)\left(|u-v|+\left|u^{\prime}-v^{\prime}\right|\right),
$$

and that

$$
\int_{\mathrm{I}} \operatorname{sk}(s) d s<\infty .
$$

In dealing with a more general type of equation than $(1.1),[11,17]$ clarify much of the situation for solutions $u(t)=c t+o(t)$. However, in contrast to these and most other investigations, our approach in this section demands nothing from the function $F$ in terms of monotonocity or its sign. Before giving some lemmas and the necessary proofs, we present the main results of this section.

THEOREM 3.2. Under Condition 3.1, suppose that for some $c \in \mathbb{R}$,

$$
\int_{\mathrm{I}}|F(s, c s, c)| d s<\infty
$$

Then any solution $u(t)$ to (1.1) satisfies

$$
\lim _{t \rightarrow \infty} \frac{u(t)}{t}=\lim _{t \rightarrow \infty} u^{\prime}(t) \in \mathbb{R} .
$$


Conversely, if there is such a solution, then any $c \in \mathbb{R}$ satisfies

$$
\sup _{t \in \mathrm{I}}\left|\int_{t_{0}}^{t} F(s, c s, c) d s\right|<\infty .
$$

COROllary 3.3. Under Condition 3.1, if $F(t, 0,0)$ is of constant sign for large $t$, then either all or none of the solutions to (1.1) satisfy $\lim _{t \rightarrow \infty} \frac{u(t)}{t}=\lim _{t \rightarrow \infty} u^{\prime}(t) \in \mathbb{R}$ according to whether $\int_{\mathrm{I}}|F(s, 0,0)| d s$ is finite or infinite, respectively.

REMARK 3.4. It is found [11] that the condition $\int_{\mathrm{I}} F(t,-c s,-c) d s<\infty$, for some $c>0$, is equivalent to $\lim _{t \rightarrow \infty} u^{\prime}(t)=$ const. $<0$, for some solution $u$. In [11] it is assumed that $F$ is positive and non-decreasing in the last two variables, whereas Condition 3.1 is not supposed. Though the results are similar, [11] and the present investigation cover different cases, and our result guarantees that all solutions are of the desired form. In [14] there are conditions guaranteeing that all solutions are of this form. There is, however, no characterization as above.

For an easy overview, the proofs will be carried out with the help of two separate lemmas, clarifying the main ideas. Once this is done, what essentially remains is an application of the Banach fixed point theorem.

LEMMA 3.5. Under Condition 3.1,

$$
\sup _{t \in \mathrm{I}}\left|\int_{t_{0}}^{t} F\left(s, u(s), u^{\prime}(s)\right) d s\right|
$$

is finite for some $u \in \mathcal{X}$ exactly if it is finite for all $u \in \mathcal{X}$. The same is true for $\int_{\mathrm{I}}\left|F\left(s, u(s), u^{\prime}(s)\right)\right| d s$.

Proof. Let $u, v \in \mathcal{X}$. Then

$$
|u(s)-v(s)|+\left|u^{\prime}(s)-v^{\prime}(s)\right| \leq(1+s)\|u-v\|_{\mathcal{X}} .
$$

Using this, and the fact that $\operatorname{sk}(s)$ is integrable by hypothesis, we conclude that there is a constant $C_{k}$, depending only on $k(t)$, such that

$$
\begin{aligned}
& \left|\int_{t_{0}}^{t} F\left(s, u(s), u^{\prime}(s)\right) d s\right| \\
& \quad \leq \int_{\mathrm{I}}\left|F\left(s, u(s), u^{\prime}(s)\right)-F\left(s, v(s), v^{\prime}(s)\right)\right| d s+\left|\int_{t_{0}}^{t} F\left(s, v(s), v^{\prime}(s)\right) d s\right| \\
& \quad \leq \int_{\mathrm{I}} k(s)\left(|u(s)-v(s)|+\left|u^{\prime}(s)-v^{\prime}(s)\right|\right) d s+\left|\int_{t_{0}}^{t} F\left(s, v(s), v^{\prime}(s)\right) d s\right| \\
& \quad \leq C_{k}\|u-v\|_{\mathcal{X}}+\left|\int_{t_{0}}^{t} F\left(s, v(s), v^{\prime}(s)\right) d s\right| .
\end{aligned}
$$

The calculation for $\int_{\mathrm{I}}\left|F\left(s, u(s), u^{\prime}(s)\right)\right| d s$ is similar.

REMARK 3.6. Lemma 3.5 enables us to consider any function in $\mathcal{X}$ to determine the finiteness of $\int_{\mathrm{I}}\left|F\left(s, u(s), u^{\prime}(s)\right)\right| d s$ and (3.4). For consistency with previous work, we choose to consider the lines through the origin $\{u \in \mathcal{X}: u(s)=c s, c \in \mathbb{R}\}$. 
Lemma 3.7. Under Condition 3.1, suppose that, for some $c \in \mathbb{R}$,

$$
\int_{\mathrm{I}}|F(s, c s, c)| d s<\infty .
$$

Then, for any initial data $u_{0}, u_{0}^{\prime} \in \mathbb{R}$, the map $T: \mathcal{X} \rightarrow \mathcal{X}$ defined by

$$
T u(t) \equiv u_{0}^{\prime}\left(t-t_{0}\right)+u_{0}+\int_{t_{0}}^{t}(s-t) F\left(s, u(s), u^{\prime}(s)\right) d s
$$

is a contraction with respect to the distance $\|\cdot\|_{\mathcal{X}}^{\varphi}$ for a suitable $\varphi$.

Proof. Put

$$
\varphi(t) \equiv \exp \left(3 \int_{t_{0}}^{t}(s+1) k(s) d s\right), \quad t \in \mathrm{I},
$$

and let $\|\cdot\|_{\mathcal{X}}^{\varphi}$ be an equivalent norm on $\mathcal{X}$ as in Remark 2.1. Note that, in view of Condition 3.1, $\varphi \geq 1$, is bounded, and has a non-negative derivative. For any $t_{1}, t_{2} \in \mathrm{I}$, we have

$$
\begin{aligned}
&\left|\frac{T u\left(t_{1}\right)-T v\left(t_{1}\right)}{\left(t_{1}+1\right) \varphi\left(t_{1}\right)}\right|+\left|\frac{(T u)^{\prime}\left(t_{2}\right)-(T v)^{\prime}\left(t_{2}\right)}{\varphi\left(t_{2}\right)}\right| \\
& \leq \frac{1}{\left(t_{1}+1\right) \varphi\left(t_{1}\right)} \int_{t_{0}}^{t_{1}}\left(t_{1}-s\right)\left|F\left(s, u(s), u^{\prime}(s)\right)-F\left(s, v(s), v^{\prime}(s)\right)\right| d s \\
&+\frac{1}{\varphi\left(t_{2}\right)} \int_{t_{0}}^{t_{2}}\left|F\left(s, u(s), u^{\prime}(s)\right)-F\left(s, v(s), v^{\prime}(s)\right)\right| d s \\
& \leq \frac{1}{\left(t_{1}+1\right) \varphi\left(t_{1}\right)} \int_{t_{0}}^{t_{1}}\left(t_{1}-s\right) k(s) \varphi(s) \frac{|u(s)-v(s)|+\left|u^{\prime}(s)-v^{\prime}(s)\right|}{\varphi(s)} d s \\
&+\frac{1}{\varphi\left(t_{2}\right)} \int_{t_{0}}^{t_{2}} k(s) \varphi(s) \frac{|u(s)-v(s)|+\left|u^{\prime}(s)-v^{\prime}(s)\right|}{\varphi(s)} d s \\
&= \frac{1}{\varphi\left(t_{1}\right)} \int_{t_{0}}^{t_{1}} \frac{\varphi^{\prime}(s)}{3} \frac{\left(t_{1}-s\right)|u(s)-v(s)|+\left|u^{\prime}(s)-v^{\prime}(s)\right|}{\left(t_{1}+1\right)} d s \\
&+\frac{1}{\varphi\left(t_{2}\right)} \int_{t_{0}}^{t_{2}} \frac{\varphi^{\prime}(s)}{3} \frac{|u(s)-v(s)|+\left|u^{\prime}(s)-v^{\prime}(s)\right|}{(s+1) \varphi(s)} d s \\
& \leq \frac{\|u-v\|_{\mathcal{X}}^{\varphi}}{3}\left(\int_{t_{0}}^{t_{1}} \frac{\varphi^{\prime}(s)}{\varphi\left(t_{1}\right)} d s+\int_{t_{0}}^{t_{2}} \frac{\varphi^{\prime}(s)}{\varphi\left(t_{2}\right)} d s\right) \\
&= \frac{\|u-v\|_{\mathcal{X}}^{\varphi}}{3}\left(\frac{\varphi\left(t_{1}\right)-\varphi\left(t_{0}\right)}{\varphi\left(t_{1}\right)}+\frac{\varphi\left(t_{2}\right)-\varphi\left(t_{0}\right)}{\varphi\left(t_{2}\right)}\right) \leq \frac{2\|u-v\|_{\mathcal{X}}^{\varphi}}{3} .
\end{aligned}
$$

For $v(t)=c t$, (3.5) yields that $T v \in \mathcal{X}$. Then $\|T u\|_{\mathcal{X}}^{\varphi} \leq\|T u-T v\|_{\mathcal{X}}^{\varphi}+\|T v\|_{\mathcal{X}}^{\varphi}$ $<\|u-v\|_{\mathcal{X}}^{\varphi}+\|T v\|_{\mathcal{X}}^{\varphi}<\infty$, for any $u \in \mathcal{X}$, so that $T$ is a well-defined contractive $\operatorname{map} \mathcal{X} \rightarrow \mathcal{X}$ in the metric given by $\|\cdot\|_{\mathcal{X}}^{\varphi}$.

Proof of Theorem 3.2. The function $v(t) \equiv c t \in \mathcal{X}$, so the assumptions of Lemma 3.7 are fulfilled. The Banach fixed point theorem yields a fixed point $u=T u \in \mathcal{X}$. Differentiating shows that such a fixed point is a solution to (1.1), and $u^{\prime}(t)=u_{0}^{\prime}-\int_{t_{0}}^{t} F\left(s, u(s), u^{\prime}(s)\right) d s$. By (3.3) and Lemma 3.5 the integral is absolutely 
convergent, and hence $u^{\prime}(t)$ has a limit, say $c$, as $t \rightarrow \infty$. Also,

$$
\frac{u(t)}{t}-u^{\prime}(t)=\frac{1}{t}\left(u_{0}-u_{0}^{\prime} t_{0}+\int_{t_{0}}^{t} s F\left(s, u(s), u^{\prime}(s)\right) d s\right),
$$

so that $u(t) / t \rightarrow c$ as $t \rightarrow \infty$, in view of Lebesgue's dominated convergence theorem.

Conversely, let $u$ be a solution of (1.1) with bounded derivative. Since any solution is a fixed point with respect to $T$, for some initial data $u_{0}, u_{0}^{\prime}$, we have that

$$
\left|\int_{t_{0}}^{t} F\left(s, u(s), u^{\prime}(s)\right) d s\right|=\left|u^{\prime}(t)-u_{0}^{\prime}\right|<\infty .
$$

Lemma 3.5 then implies that this inequality holds for any $v \in \mathcal{X}$, and in particular for $v(t) \equiv c t$.

Proof of Corollary 3.3. This follows from Theorem 3.2 and Lemma 3.5.

4. Asymptotically linear solutions with fixed initial data. We shall now study solutions of (1.1) that fully satisfy (1.2). Solutions asymptotic to lines have earlier been investigated, e.g. in $[\mathbf{9}, \mathbf{1 2}, \mathbf{1 4}, \mathbf{1 5}, \mathbf{1 6}]$. The papers $[\mathbf{9}, \mathbf{1 2}, \mathbf{1 6}]$ deal with the case when $F=F(t, u)$ does not depend on $u^{\prime}$. The paper [14] is, among other things, concerned with solutions $u(t)=c t+m+o(1)$, which in general is a slightly larger class than (1.2). Their assumptions are different, and in relevant cases we compare our results to theirs. The very recent contribution [15] is a general treatise on solutions asymptotic to polynomials, but again the assumptions are different from ours. Whereas in Section 5 the focus will be on the asymptote $c t+m$, instead we will now fix the initial data and ask if the corresponding solution is of form (1.2). Theorem 4.2 will give conditions guaranteeing that this is the case, and Corollary 4.3 gives criteria for such a solution to be positive and non-decreasing. An analogue of the suffiency part of Corollary 3.3, Corollary 4.5 then presents a condition for all solutions to be asymptotic to straight lines.

In this section, a monotonicity property will be imposed on $F(t, \cdot, \cdot)$. On the other hand there are still no sign conditions on $F$ and Condition 3.1 will be weakened to the following generalized version:

CONDITION 4.1. Let

$$
\mathcal{C}=\left\{\left(t, u, u^{\prime}\right) \in \mathrm{I} \times \mathbb{R} \times \mathbb{R}:|u| \leq c(t+1),\left|u^{\prime}\right| \leq c\right\} .
$$

Suppose that there exists a continuous function $k: \mathrm{I} \rightarrow[0, \infty)$, such that for any $\left(t, u, u^{\prime}\right),\left(t, v, v^{\prime}\right) \in \mathcal{C}$,

$$
\left|F\left(t, u, u^{\prime}\right)-F\left(t, v, v^{\prime}\right)\right| \leq k(t)\left(|u-v|+\left|u^{\prime}-v^{\prime}\right|\right),
$$

with $\int_{\mathrm{I}} s k(s) d s<\infty$, and that for any $\left(t, u, u^{\prime}\right) \in \mathcal{C}$, we have

$$
\left|F\left(t, u, u^{\prime}\right)\right| \leq|F(t, c(t+1), c)| .
$$

Whereas Condition 3.1 guarantees that all solutions to (1.1) are globally defined in time, Condition 4.1 also accommodates equations with solutions that blow-up in finite time. 
THEOREM 4.2. If Condition 4.1 holds for some $c \geq 0$, and

$$
\int_{\mathrm{I}}|s F(s, c(s+1), c)| d s \leq c-\left|u_{0}\right|, \quad \int_{\mathrm{I}}|F(s, c(s+1), c)| d s \leq c-\left|u_{0}^{\prime}\right|,
$$

then there exists a solution $u$ to (1.1) with $u\left(t_{0}\right)=u_{0}, u^{\prime}\left(t_{0}\right)=u_{0}^{\prime}$, and $u$ is asymptotic to a straight line, i.e. (1.2) holds for some constants.

COROLlaRY 4.3. If Condition 4.1 holds for some $c \geq 0$, and

$$
\int_{\mathrm{I}}|s F(s, c(s+1), c)| d s \leq c, \quad \int_{\mathrm{I}}|F(s, c(s+1), c)| d s \leq c / 2,
$$

then there exists a positive non-decreasing solution $u$ to $(1.1)$ with $u\left(t_{0}\right)=0, u^{\prime}\left(t_{0}\right)=c / 2$, and $u$ is asymptotic to a straight line, i.e. (1.2) holds for some constants.

REMARK 4.4. We have found no analogue of Corollary 4.3, but one can recognize part of (4.3) from other results. E.g. in [4], dealing with the special case $F=F(t, u)$, $\int_{\mathrm{I}} g(s, c s) d s<c / 2$ is the sufficient condition for the existence of a positive solution with $u(0)=0$ and $u(t) / t=c / 2+o(t)$ as $t \rightarrow \infty$, provided $|F(t, u)| \leq g(t,|u|)$ where $g(t, \cdot)$ is non-decreasing. Note that the solution presented in Corollary 4.3 satisfies the much stronger property (1.2), hence the stronger condition (4.3).

Corollary 4.5. Let $\mathrm{I} \subseteq[1, \infty), c_{0} \geq 0$, and suppose that Condition 4.1 holds for all $c \geq c_{0}$. If there exists $\alpha \in(0,1)$ such that

$$
\int_{\mathrm{I}}|s F(s, c(s+1), c)| d s \leq \alpha c, \quad \text { whenever } \quad c \geq c_{0},
$$

then all solutions to (1.1) are asymptotic to straight lines, i.e. for every solution the relation (1.2) holds for some constants.

REMARK 4.6. [14] too delivers criteria for every initial data $\left(t_{0}, u_{0}, u_{0}^{\prime}\right) \in[1, \infty) \times$ $\mathbb{R} \times \mathbb{R}$ to correspond to a solution asymptotic to a line, in the slightly weaker sense $u(t)=c t+m+o(1)$ as $t \rightarrow \infty$. They require that $\left|F\left(t, u, u^{\prime}\right)\right| \leq k(t)\left[p_{1}(|u| / t)+\right.$ $\left.p_{2}\left(\left|u^{\prime}\right|\right)\right]$, where $p_{1}, p_{2}$ are positive, non-decreasing, and $k$ satisfies $\int_{\mathrm{I}} s k(s) d s<\infty$. Also, $\int_{\mathrm{I}}\left(p_{1}(s)+p_{2}(s)\right)^{-1} d s=\infty$ must hold.

REMARK 4.7. The requirement $I \subseteq[1, \infty)$ should not be considered a restriction. It is imposed only to simplify the condition (4.4). Any solution to (1.1) can be extended to the left at least as long as Condition 3.1 holds.

Proof of Theorem 4.2. We will consider the complete metric space $\mathcal{X}_{c}$ defined by (2.4), where $c$ is given by assumption. For any $u \in \mathcal{X}_{c}$, we have that $\left|F\left(t, u(t), u^{\prime}(t)\right)\right| \leq$ $|F(t, c(t+1), c)|$. Consequently

$$
\int_{\mathrm{I}}\left|s F\left(s, u(s), u^{\prime}(s)\right)\right| d s \leq c-\left|u_{0}\right|, \quad \int_{\mathrm{I}}\left|F\left(s, u(s), u^{\prime}(s)\right)\right| d s \leq c-\left|u_{0}^{\prime}\right| .
$$

The map $T: \mathcal{X}_{c} \rightarrow \mathcal{X}$ presented in Lemma 3.7 is well-defined, and we will show that it is actually a contraction $\mathcal{X}_{c} \rightarrow \mathcal{X}_{c}$. In view of (4.5), and by the triangle inequality, $|T u(t)| \leq(t+1) c$ and $\left|(T u)^{\prime}(t)\right| \leq c$. Hence $T$ maps $\mathcal{X}_{c}$ into $\mathcal{X}_{c}$. As for what concerns the contraction, the proof of Lemma 3.7 holds in every detail since Condition 4.1 guarantees that (3.1-3.2) hold for any functions in $\mathcal{X}_{c}$. The Banach fixed point 
theorem implies the existence of a unique fixed point $u=T u \in \mathcal{X}_{c}$, and consequently a corresponding solution to (1.1). By the absolute convergence given by (4.5) it follows that

$$
\begin{aligned}
& \lim _{t \rightarrow \infty}\left|u(t)-\left(u_{0}-u_{0}^{\prime} t_{0}+\int_{\mathrm{I}} s F\left(s, u(s), u^{\prime}(s)\right) d s\right)-\left(u_{0}^{\prime}-\int_{\mathrm{I}} F\left(s, u(s), u^{\prime}(s)\right) d s\right) t\right| \\
& =\lim _{t \rightarrow \infty}\left|u^{\prime}(t)-u_{0}^{\prime}+\int_{\mathrm{I}} F\left(s, u(s), u^{\prime}(s)\right) d s\right|=0,
\end{aligned}
$$

so that (1.2) holds.

Proof of Corollary 4.3 This is Theorem 4.2 with $u_{0}=0, u_{0}^{\prime}=c / 2$. Then the derivative $u_{0}^{\prime}-\int_{t_{0}}^{t} F\left(s, u(s), u^{\prime}(s)\right) d s \geq 0$, since

$$
\left|\int_{t_{0}}^{t} F\left(s, u(s), u^{\prime}(s)\right) d s\right| \leq \int_{\mathrm{I}}|F(s, c(s+1), c)| d s \leq u_{0}^{\prime} .
$$

Proof of Corollary 4.5

$$
\int_{\mathrm{I}}|F(s, c(s+1), c)| d s \leq \int_{\mathrm{I}}|s F(s, c(s+1), c)| d s \leq \alpha c .
$$

It follows that for any pair $\left(u_{0}, u_{0}^{\prime}\right) \in \mathbb{R} \times \mathbb{R}$ we can find $c$ so large that the assumptions of Theorem 4.2 are fulfilled. By classical uniqueness theorems for ordinary differential equations we get all solutions to (1.1) on I by letting $\left(u_{0}, u_{0}^{\prime}\right)$ range over $\mathbb{R} \times \mathbb{R}$.

5. Asymptotically linear solutions with fixed asymptote. Turning our focus towards solutions asymptotic to a given line, we present in this section two different approaches. Theorem 5.2 gives sufficient, respectively necessary, conditions for the existence of a unique solution asymptotic to $c t+m$ in a slightly stricter sense than (1.2). In the light of $F(t, 0,0) \geq 0$ (or $\leq 0$ ) for $t$ large, Corollary 5.4 characterizes these solutions. While these results both make use of Condition 3.1 and the Banach fixed point theorem, Theorem 5.6 is an application of the Schauder fixed point theorem. Condition 3.1 is not required, but instead there is a monotonicity assumption on $F(t, \cdot, \cdot)$.

REMARK 5.1. While here we examine linear asymptotic behaviour, the recent investigation [7] is devoted to finding conditions guaranteeing the existence of solutions that approach an arbitrary given function. Due to the generality of the question posed, that paper deals with first order equations.

TheOREM 5.2. Under Condition 3.1, let c, $m \in \mathbb{R}$, and suppose that

$$
\int_{\mathrm{I}}|s F(s, c s, c)| d s<\infty .
$$

Then there exists a unique solution $u \in C_{c, m}^{1}(\mathrm{I})$ to (1.1) so that (1.2) holds and $\mid t u^{\prime}(t)-$ $c t \mid \rightarrow 0$ as $t \rightarrow \infty$. Conversely, if there is such a solution, then

$$
\sup _{t \in \mathrm{I}}\left|\int_{t_{0}}^{t} s F(s, c s, c) d s\right|+\sup _{t \in \mathrm{I}}\left|\int_{t_{0}}^{t} F(s, c s, c) d s\right|<\infty .
$$


REMARK 5.3. Even if $|s F(s, c s, c)|$ is integrable for all $c \in \mathbb{R}$, we cannot conclude from Theorem 5.2 that all solutions are of form (1.2).

Corollary 5.4. Under Condition 3.1, let c, $m \in \mathbb{R}$ and suppose that $F(s, c s, c)$ is of constant sign for large $s$. Then the existence of a unique solution $u \in C_{c, m}^{1}$ (I) to (1.1) so that (1.2) holds and $\lim _{t \rightarrow \infty}\left|t u^{\prime}(t)-c t\right|=0$ is equivalent to

$$
\int_{\mathrm{I}}|s F(s, c s, c)| d s<\infty .
$$

REMARK 5.5. The investigation [14] gives sufficient conditions for the existence of solutions $u(t)=c t+m+o(t)$ for all $c, m \in \mathbb{R}$. These conditions are identical to the ones referred to in Remark 4.6. Also in [15] sufficient conditions are given, based upon a generalization of the conditions in [14]. Our result is different because it characterizes the equations that have solutions satisfying (1.2) and $\lim _{t \rightarrow \infty}\left|t u^{\prime}-c t\right|=0$, for certain $c, m \in \mathbb{R}$.

THEOREM 5.6. Let $\mathrm{I} \subseteq[1, \infty), c, m \in \mathbb{R}$, and define $a(t) \equiv|c|(t+1)+|m|$. Suppose that for $\left(t, u, u^{\prime}\right) \in \mathrm{I} \times \mathbb{R} \times \mathbb{R}$ such that $|u| \leq a(t),\left|u^{\prime}\right| \leq 2|c|$ we have

$$
\left|F\left(t, u, u^{\prime}\right)\right| \leq|F(t, a(t), 2|c|)| .
$$

If $F(t, a(t), 2|c|)$ is bounded, and

$$
\int_{\mathrm{I}}|s F(s, a(s), 2|c|)| d s \leq|c|,
$$

then there exists a solution $u(t)$ to (1.1) on I, asymptotic to $c t+m$ so that (1.2) holds.

As in Section 3, two lemmas will help us present the proofs of Theorem 5.2 and Corollary 5.4. The proof of Theorem 5.6 is delayed until the end of the section.

LEMMA 5.7. Under Condition 3.1,

$$
\sup _{t \in \mathrm{I}}\left|\int_{t_{0}}^{t} s F\left(s, u(s), u^{\prime}(s)\right) d s\right|
$$

is finite for $u(t) \equiv$ ct exactly if it is finite for all $u \in\left\{C_{c, m}^{1}(\mathrm{I})\right\}_{m \in \mathbb{R}}$. The same holds true for $\int_{\mathrm{I}}\left|s F\left(s, u(s), u^{\prime}(s)\right)\right| d s$.

Proof. For any $\varepsilon>0$ and $u \in C_{c, m}^{1}(\mathrm{I})$, we can find $t_{\varepsilon} \geq t_{0}$ such that $|u(t)-c t|+$ $\left|u^{\prime}(t)-c\right|<|m|+\varepsilon$ whenever $t \geq t_{\varepsilon}$. As a consequence, Condition 3.1 implies that for any $u \in C_{c, m}^{1}(\mathrm{I})$,

$$
\begin{aligned}
& \left|\int_{t_{0}}^{t} s F\left(s, u(s), u^{\prime}(s)\right) d s\right| \leq \int_{\mathrm{I}} s k(s)\left(|u(s)-c s|+\left|u^{\prime}(s)-c\right|\right) d s+\left|\int_{t_{0}}^{t} s F(s, c s, c) d s\right| \\
& \quad \leq \int_{t_{0}}^{t_{\varepsilon}} s k(s)\left(|u(s)-c s|+\left|u^{\prime}(s)-c\right|\right) d s+\int_{t_{\varepsilon}}^{\infty} s k(s)(|m|+\varepsilon) d s+\left|\int_{t_{0}}^{t} s F(s, c s, c) d s\right| .
\end{aligned}
$$

We could just as well reverse the roles of $v(t)=c t$ and $u(t)$. Hence all of $\left\{C_{c, m}^{1}(\mathrm{I})\right\}_{m \in \mathbb{R}}$ is equivalent to $v(t)=c t$ in the sense of finiteness of (5.3). The proof of the second statement is identical. 
LEMMA 5.8. Under condition 3.1, let $c, m \in \mathbb{R}$, and suppose that

$$
\int_{\mathrm{I}}|s F(s, c s, c)| d s<\infty
$$

Then the map $S: C_{c, m}^{1}(\mathrm{I}) \rightarrow C_{c, m}^{1}(\mathrm{I})$, defined by

$$
S u(t) \equiv c t+m-\int_{t}^{\infty}(s-t) F\left(s, u(s), u^{\prime}(s)\right) d s, \quad t \in \mathrm{I},
$$

is a contraction with respect to the distance $\|\cdot\|_{1}^{\varphi}$ for a suitable $\varphi$.

Proof. Define the auxilliary function

$$
\varphi(t) \equiv \exp \left(3 \int_{t}^{\infty}\left(s+1-t_{0}\right) k(s) d s\right), \quad t \in \mathrm{I},
$$

and consider the complete metric space $C_{c, m}^{1}(\mathrm{I})$ with the metric induced by $\|\cdot\|_{1}^{\varphi}$ (cf. Remark 2.1). By Condition 3.1, $\varphi(t)$ is bounded, $\varphi^{\prime}(t) \leq 0$ and $\lim _{t \rightarrow \infty} \varphi(t)=1$. First note that (5.5) and Lemma 5.7 imply that $|S u(t)-c t-m| \rightarrow 0$ and $\mid(S u)^{\prime}(t)-$ $c \mid \rightarrow 0$ as $t \rightarrow \infty$, so that $S u \in C_{c, m}^{1}(\mathrm{I})$ whenever $u \in C_{c, m}^{1}(\mathrm{I})$. Now, take any $t_{1}, t_{2} \in \mathrm{I}$. Then

$$
\begin{aligned}
\left|\frac{S u\left(t_{1}\right)-S v\left(t_{1}\right)}{\varphi\left(t_{1}\right)}\right|+\left|\frac{(S u)^{\prime}\left(t_{2}\right)-(S v)^{\prime}\left(t_{2}\right)}{\varphi\left(t_{2}\right)}\right| & \frac{1}{\varphi\left(t_{1}\right)} \int_{t_{1}}^{\infty}\left(s-t_{1}\right)\left|F\left(s, u(s), u^{\prime}(s)\right)-F\left(s, v(s), v^{\prime}(s)\right)\right| d s \\
& +\frac{1}{\varphi\left(t_{2}\right)} \int_{t_{2}}^{\infty}\left|F\left(s, u(s), u^{\prime}(s)\right)-F\left(s, v(s), v^{\prime}(s)\right)\right| d s \\
\leq & \frac{1}{\varphi\left(t_{1}\right)} \int_{t_{1}}^{\infty}\left(s-t_{1}\right) k(s) \varphi(s) \frac{|u(s)-v(s)|+\left|u^{\prime}(s)-v^{\prime}(s)\right|}{\varphi(s)} d s \\
& +\frac{1}{\varphi\left(t_{2}\right)} \int_{t_{2}}^{\infty} k(s) \varphi(s) \frac{|u(s)-v(s)|+\left|u^{\prime}(s)-v^{\prime}(s)\right|}{\varphi(s)} d s \\
= & \frac{1}{\varphi\left(t_{1}\right)} \int_{t_{1}}^{\infty} \frac{-\varphi^{\prime}(s)}{3} \frac{\left(s-t_{1}\right)}{\left(s+1-t_{0}\right)} \frac{|u(s)-v(s)|+\left|u^{\prime}(s)-v^{\prime}(s)\right|}{\varphi(s)} d s \\
& +\frac{1}{\varphi\left(t_{2}\right)} \int_{t_{2}}^{\infty} \frac{-\varphi^{\prime}(s)}{3\left(s+1-t_{0}\right)} \frac{|u(s)-v(s)|+\left|u^{\prime}(s)-v^{\prime}(s)\right|}{\varphi(s)} d s \\
\leq & \frac{\|u-v\|_{1}^{\varphi}}{3}\left(\int_{t_{1}}^{\infty} \frac{-\varphi^{\prime}(s)}{\varphi\left(t_{1}\right)} d s+\int_{t_{2}}^{\infty} \frac{-\varphi^{\prime}(s)}{\varphi\left(t_{2}\right)} d s\right) \\
= & \frac{\|u-v\|_{1}^{\varphi}}{3}\left(\frac{\varphi\left(t_{1}\right)-1}{\varphi\left(t_{1}\right)}+\frac{\varphi\left(t_{2}\right)-1}{\varphi\left(t_{2}\right)}\right) \leq \frac{2\|u-v\|_{1}^{\varphi}}{3} .
\end{aligned}
$$

Hence $S$ is a contraction with respect to $\|\cdot\|_{1}^{\varphi}$.

Proof of Theorem 5.2. Lemma 5.8 and the Banach fixed point theorem imply the existence of a unique function $u \in C_{c, m}^{1}$ (I) that satisfies $S u=u$. Then $u$ satisfies (1.1) so that it is the unique solution on I satisfying (1.2). It is easily seen from (5.6) that $\left|t u^{\prime}(t)-c t\right| \rightarrow 0$ as $t \rightarrow \infty$. 
Conversely, every solution to (1.1) on I satisfies

$$
u(t)=u_{0}^{\prime} t+u_{0}+\int_{t_{0}}^{t}(s-t) F\left(s, u(s), u^{\prime}(s)\right) d s, \quad t \in \mathrm{I},
$$

for some $u_{0}, u_{0}^{\prime}$. We have

$$
u^{\prime}(t)=u_{0}^{\prime}-\int_{t_{0}}^{t} F\left(s, u(s), u^{\prime}(s)\right) d s,
$$

so that by the asymptotic behaviour of $u^{\prime}(t)$,

$$
\lim _{t \rightarrow \infty} \int_{t_{0}}^{t} F\left(s, u(s), u^{\prime}(s)\right) d s=u_{0}^{\prime}-c .
$$

Combining this with the asymptotic behaviour of $u(t)$, taking into account that $\lim _{t \rightarrow \infty}\left|t u^{\prime}(t)-c t\right|=0$, we see that

$$
\lim _{t \rightarrow \infty} \int_{t_{0}}^{t} s F\left(s, u(s), u^{\prime}(s)\right) d s=m-u_{0} .
$$

Lemma 5.7 almost completes the proof. One need just use (5.4), reversing the roles of $u$ and $c s$, and taking the $\lim \sup _{t \rightarrow \infty}$, with and without the $s$ in front of $F$.

Proof of Corollary 5.4. Since $F(s, c s, c)$ does not change sign on a half-axis, the finiteness of $\sup _{t \in \mathrm{I}}\left|\int_{t_{0}}^{t} s F(s, c s, c) d s\right|$ implies finiteness of $\int_{\mathrm{I}}|s F(s, c s, c)| d s$, yielding $\int_{\mathrm{I}}|F(s, c s, c)| d s<\infty$.

Proof of Theorem 5.6 The norm

$$
\|u\|_{1}^{\max } \equiv \max \left\{\sup _{t \in \mathrm{I}}|u(t)|, \sup _{t \in \mathrm{I}}\left|u^{\prime}(t)\right|\right\}
$$

induces a metric equivalent to that of the ordinary extended supremum norm $\|\cdot\|_{1}$. We shall consider the strip of functions

$$
\mathcal{C} \equiv\left\{u \in C^{1}(\mathrm{I}):\|u-c t-m\|_{1}^{\max } \leq|c|\right\} .
$$

Note that $\mathcal{C}$ is a closed, bounded, convex, and nonempty subset of the Banach space $\left(C^{1}(\mathrm{I}),\|\cdot\|_{\mathcal{X}}\right)$. We will make use of the map $S$ defined in Lemma 5.8, which we will show is a continuous map $\mathcal{C} \rightarrow \mathcal{C}$ with a pre-compact image. Let $u \in \mathcal{C}$. Then $|u(t)| \leq$ $|c|(t+1)+|m|$, and $\left|u^{\prime}(t)\right| \leq 2|c|$, so that, by assumption,

$$
\begin{aligned}
|S u(t)-c t-m| & \leq \int_{t}^{\infty}\left|(s-t) F\left(s, u(s), u^{\prime}(s)\right)\right| d s \leq \int_{t}^{\infty}\left|s F\left(s, u(s), u^{\prime}(s)\right)\right| d s \\
& \leq \int_{\mathrm{I}}|s F(s,|c|(s+1)+|m|, 2|c|)| d s \leq|c|,
\end{aligned}
$$

and

$$
\begin{aligned}
\left|(S u)^{\prime}(t)-c\right| & \leq \int_{t}^{\infty}\left|F\left(s, u(s), u^{\prime}(s)\right)\right| d s \\
& \leq \int_{\mathrm{I}}|F(s,|c|(s+1)+|m|, 2|c|)| d s \leq|c|,
\end{aligned}
$$

implying that $S u \in \mathcal{C}$. 
We need to show that $\mathcal{S}$ is a continuous map $\mathcal{C} \rightarrow \mathcal{C}$ in the metric induced by $\|\cdot\|_{\mathcal{X}}$. Let $\varepsilon>0$. By hypothesis, there exists $t_{\varepsilon}>1+t_{0}$, such that

$$
\int_{t_{\varepsilon}}^{\infty}\left|s F\left(s, u(s), u^{\prime}(s)\right)\right| d s<\varepsilon / 8,
$$

for all $u \in \mathcal{C}$. On the cube

$$
K \equiv\left[t_{0}, t_{\varepsilon}\right] \times\left[-|c|\left(1+t_{\varepsilon}\right)-|m|,|c|\left(1+t_{\varepsilon}\right)+|m|\right] \times[-2|c|, 2|c|],
$$

the function $F$ is uniformly continuous, so presuming that $\left(s, u, u^{\prime}\right),\left(s, v, v^{\prime}\right) \in K$, there exists a $\delta>0$ with

$$
\left|F\left(s, u, u^{\prime}\right)-F\left(s, v, v^{\prime}\right)\right|<\frac{\varepsilon}{4\left(t_{\varepsilon}-t_{0}\right)^{2}}, \quad \text { for } \quad|u-v|+\left|u^{\prime}-v^{\prime}\right|<\delta .
$$

For functions $u, v \in \mathcal{C}$ this will be the case on $\left[t_{0}, t_{\varepsilon}\right]$ if

$$
\|u-v\|_{\mathcal{X}}<\frac{\delta}{1+t_{\varepsilon}}=\eta_{\varepsilon} .
$$

So whenever this inequality holds, we have that

$$
\begin{aligned}
|S u(t)-S v(t)| \leq & \int_{t}^{\infty}(s-t)\left|F\left(s, u(s), u^{\prime}(s)\right)-F\left(s, v(s), v^{\prime}(s)\right)\right| d s \\
\leq & \int_{t}^{t_{\varepsilon}}(s-t)\left|F\left(s, u(s), u^{\prime}(s)\right)-F\left(s, v(s), v^{\prime}(s)\right)\right| d s \\
& +\int_{t_{\varepsilon}}^{\infty} s\left(\left|F\left(s, u(s), u^{\prime}(s)\right)\right|+\left|F\left(s, v(s), v^{\prime}(s)\right)\right|\right) d s<\varepsilon / 2
\end{aligned}
$$

and

$$
\begin{aligned}
\left|(S u)^{\prime}(t)-(S v)^{\prime}(t)\right| \leq & \int_{t}^{\infty}\left|F\left(s, u(s), u^{\prime}(s)\right)-F\left(s, v(s), v^{\prime}(s)\right)\right| d s \\
\leq & \int_{t}^{t_{\varepsilon}}\left|F\left(s, u(s), u^{\prime}(s)\right)-F\left(s, v(s), v^{\prime}(s)\right)\right| d s \\
& +\int_{t_{\varepsilon}}^{\infty}\left(\left|F\left(s, u(s), u^{\prime}(s)\right)\right|+\left|F\left(s, v(s), v^{\prime}(s)\right)\right|\right) d s<\varepsilon / 2 .
\end{aligned}
$$

Hence $\|S u-S v\|_{\mathcal{X}}<\varepsilon$ whenever $\|u-v\|_{\mathcal{X}}<\eta_{\varepsilon}$.

To prove that $S \mathcal{C}$ is relatively compact, take any sequence $\left\{u_{n}\right\}_{n} \subset \mathcal{C}, n \in \mathbb{N}$, and put $w_{n}(t) \equiv S u_{n}(t)-c t-m$. Then $\left|w_{n}(t)\right| \leq|c|$, so $\left\{w_{n}\right\}_{n}$ is uniformly bounded on $\mathrm{I}$. Furthermore, for any $t_{2} \geq t_{1} \geq t_{0}$,

$$
\begin{aligned}
\left|w_{n}\left(t_{1}\right)-w_{n}\left(t_{2}\right)\right| & =\left|\int_{t_{1}}^{\infty}\left(s-t_{1}\right) F\left(s, u_{n}(s), u_{n}^{\prime}(s)\right) d s-\int_{t_{2}}^{\infty}\left(s-t_{2}\right) F\left(s, u_{n}(s), u_{n}^{\prime}(s)\right) d s\right| \\
& \leq \int_{t_{1}}^{t_{2}}\left|\left(s-t_{1}\right) F\left(s, u_{n}(s), u_{n}^{\prime}(s)\right)\right| d s+\left(t_{2}-t_{1}\right) \int_{t_{2}}^{\infty}\left|F\left(s, u_{n}(s), u_{n}^{\prime}(s)\right)\right| d s \\
& \leq\left(t_{2}-t_{1}\right) \int_{\mathrm{I}}|F(s,|c|(s+1)+|m|, 2|c|)| d s \leq|c|\left(t_{2}-t_{1}\right),
\end{aligned}
$$


implying uniform equicontinuity of $\left\{w_{n}\right\}_{n}$. In effect, the Arzela-Ascoli Theorem yields the existence of a continuous function $w(t)$ and a subsequence $\left\{w_{n_{k}}\right\}_{n_{k}} \subset\left\{w_{n}\right\}_{n}$, such that $w_{n_{k}} \rightarrow w$ uniformly on I as $n_{k} \rightarrow \infty$.

This can be seen in the following way. On every compact interval $\mathrm{I}_{N} \equiv\left[t_{0}, N\right]$, $t_{0} \leq N \in \mathbb{N}$, we can apply the Arzela-Ascoli Theorem to conclude that there is a subsequence $\left\{w_{n_{N}}\right\}_{n_{N}}$ converging uniformly to a continuous function $w$ on $\mathrm{I}_{N}$. Then this subsequence in its turn contains a subsequence that converges uniformly to a continuous function on $\mathrm{I}_{N}$ for some larger $N$, and it follows that this limit function is just an extension of $w$, so we will call it $w$. Repeating the argument for an increasing sequence of $N$ 's shows that $w$ is globally defined on $\left[t_{0}, \infty\right)$, and there is a diagonal subsequence $\left\{w_{N_{N}}\right\}$ converging uniformly to $w$ on every compact interval. By (5.7), any $w_{n}$ in the original sequence tends to 0 as $t \rightarrow \infty$, and this convergence is uniform for all $w_{n}$. This means that for any $\varepsilon>0$ there exists a $t_{\varepsilon}$ such that

$$
\left|w_{N_{N}}(t)\right|<\varepsilon / 3, \quad \text { whenever } t \geq t_{\varepsilon} .
$$

By the triangle inequality,

$$
|w(t)| \leq\left|w(t)-w_{N_{N}}(t)\right|+\left|w_{N_{N}}(t)\right|<2 \varepsilon / 3, \quad \text { whenever } t \geq t_{\varepsilon},
$$

since for any fixed $t \geq t_{\varepsilon}$ there is an $N$ with $\left|w(t)-w_{N_{N}}(t)\right|<\varepsilon / 3$. We then fix an interval $\mathrm{I}_{\varepsilon}$ containing $t_{\varepsilon}$ and subsequently an $N_{0}$ such that

$$
\sup _{t \in \mathrm{I}_{\varepsilon}}\left|w_{N_{N}}-w\right|<\varepsilon, \quad \text { whenever } N \geq N_{0} .
$$

Taking all this into account, we see that

$$
\sup _{t \in \mathrm{I}}\left|w(t)-w_{N_{N}}(t)\right|<\varepsilon, \quad \text { whenever } N \geq N_{0},
$$

and it follows that the subsequence $\left\{w_{n_{k}}\right\}$ above could be taken to equal $\left\{w_{N_{N}}\right\}$.

It is now immediate from the definition of $w_{n}$ that $S u_{n_{k}}$ converges uniformly to $u(t)=w(t)+c t+m$ on I, and for simplicity we denote the subsequence $\left\{S u_{n_{k}}\right\}_{n_{k}}$ again by $\left\{S u_{n}\right\}_{n}$.

Now define $w_{n}^{\prime}(t) \equiv\left(S u_{n}\right)^{\prime}(t)-c$. Since $S u_{n} \in \mathcal{C}$, we have that $\left|w_{n}^{\prime}(t)\right| \leq|c|$, for all $n \in \mathbb{N}$. The fact that $\left\{w_{n}^{\prime}\right\}_{n}$ is uniformly equicontinuous follows from

$$
\begin{aligned}
\left|w_{n}^{\prime}\left(t_{1}\right)-w_{n}^{\prime}\left(t_{2}\right)\right| & \leq \int_{t_{1}}^{t_{2}}\left|F\left(s, u_{n}(s), u_{n}^{\prime}(s)\right)\right| d s \\
& \leq \int_{t_{1}}^{t_{2}}|F(s,|c|(s+1)+|m|, 2|c|)| d s \leq\left(t_{2}-t_{1}\right) B,
\end{aligned}
$$

since $F(t,|c|(t+1)+|m|, 2|c|)$ is bounded by assumption. Again, by the Arzela-Ascoli Theorem, there is a subsequence $\left\{w_{n_{k}}^{\prime}\right\}_{n_{k}}$ and a continuous function $w^{\prime}$ such that $w_{n_{k}}^{\prime} \rightarrow w^{\prime}$ uniformly on I as $n_{k} \rightarrow \infty$. The details of this argument are the same as in the above argument concerning $w_{n}$. The main ingredients are (5.8), used for large $t$, and the Arzela-Ascoli theorem, used on compact intervals. In effect, $\left(S u_{n_{k}}\right)^{\prime}$ converges uniformly to $\tilde{u}(t) \equiv w^{\prime}(t)+c$ on I. As before, we write $\left\{S u_{n}\right\}_{n}$ for the new subsequence. By the Fundamental theorem of analysis,

$$
S u_{n}(t)=S u_{n}\left(t_{0}\right)+\int_{t_{0}}^{t}\left(S u_{n}\right)^{\prime}(s) d s,
$$


and in view of the uniform convergence of both $S u_{n}$ and $\left(S u_{n}\right)^{\prime}$, taking limits yields

$$
u(t)=u\left(t_{0}\right)+\int_{t_{0}}^{t} \tilde{u}(s) d s .
$$

We conclude that $u^{\prime}=\tilde{u}$ and we have found a subsequence $\left\{S u_{n}\right\}_{n}$ with a limit in $C^{1}(\mathrm{I})$ in the sense of uniform convergence. But then $S u_{n}$ converges to this limit also in the sense of $\|\cdot\|_{\mathcal{X}}$. We have thus proved that the image $S \mathcal{C}$ is relatively compact.

The requirements of the Schauder fixed point theorem are fulfilled (cf [20]), and we have a fixed point, say $u=S u \in \mathcal{C}$. It remains only to check that (1.2) holds. This is guaranteed by (5.1) and the absolute convergence of the integral in (5.2).

6. Examples. We give here some applications of the results. Example 6.1 shows that special cases of our investigations easily yield different classic results, and Example 6.2 shows that conclusions can be drawn for the special class (1.2) even in the case of nonlinearities with fast growth rate.

EXAMPLE 6.1. According to Corollary 3.3, every solution of the linear equation

$$
u^{\prime \prime}(t)+a(t) u^{\prime}(t)+b(t) u(t)=0, \quad t \in \mathrm{I}
$$

satisfies $u(t) / t \rightarrow$ const. as $t \rightarrow \infty$ if $\int_{\mathrm{I}} s(|a(s)|+|b(s)|) d s<\infty$. Supposing this, Corollary 5.4 says that either there exists a solution of the form (1.2) with

$$
\lim _{t \rightarrow \infty}\left|t u^{\prime}(t)-c t\right|=0 \quad \text { for all } c, m \in \mathbb{R},
$$

or there exists no such solution, according to whether

$$
\int_{I}\left|s a(s)+s^{2} b(s)\right| d s
$$

is finite or not. Corollary 4.5 furthermore implies that, if $a(t), b(t) \geq 0$, and

$$
\int_{\mathrm{I}}|s a(s)+s(s+1) b(s)| d s<1,
$$

then any solution satisfies (1.2) for some constants.

EXAMPLE 6.2. For $c \geq 2$, consider the nonlinear equation

$$
u^{\prime \prime}(t)+\exp (-2 c t)\left(u^{\prime}(t)+\exp (u)\right), \quad t \in[1, \infty) \equiv \mathrm{I} .
$$

By Corollary 4.3 there exists a positive non-decreasing solution with $u(1)=0$ and $u^{\prime}(1)=c / 2$, asymptotic to a line so that (1.2) holds. By Theorem 5.6, whenever $m \leq$ $\log c$, there exists a solution asymptotic to $c t+m$ in the sense of (1.2).

Proof. By the Mean value theorem, the choice $k(t)=\exp (c-c t)$ satisfies Condition 4.1 for $c$. We have that

$$
\begin{aligned}
\int_{\mathrm{I}}|s F(s, c(s+1), c)| d s & =\int_{\mathrm{I}} s(\exp (c-c s)+c \exp (-2 c s)) d s \\
& \leq 2 \exp (c) \int_{\mathrm{I}} s \exp (-c s) d s \leq \frac{2(c+1)}{c^{2}} \leq c
\end{aligned}
$$


and

$$
\int_{\mathrm{I}}|F(s, c(s+1), c)| d s \leq 2 / c \leq c / 2
$$

so that the assumptions of Corollary 4.3 are fulfilled.

To apply Theorem 5.6, we need to verify that

$$
\int_{\mathrm{I}} s \exp (-2 c s)(2 c+\exp (c s+c+m)) d s \leq c .
$$

In view of $m \leq \log c$, and using that $2 \exp (-c) \leq 1 / 3$, the left-hand side is

$$
\begin{aligned}
& 2 c \int_{\mathrm{I}} s \exp (-2 c s) d s+\exp (c+m) \int_{\mathrm{I}} s \exp (-c s) d s \\
& \quad \leq(2 c+\exp (c+m)) \int_{\mathrm{I}} s \exp (-c s) d s \\
& \quad=(2 c+\exp (c+m)) \frac{c+1}{c^{2}} \exp (-c) \\
& \quad \leq(2 \exp (-c)+1) \frac{c+1}{c} \leq \frac{4(c+1)}{3 c} \leq c,
\end{aligned}
$$

since $c \geq 2$. Moreover, $\exp (-2 c s)(2 c+\exp (c s+c+m))$ is clearly bounded.

Acknowledgement. The author is thankful to the referee for constructive criticism and helpful suggestions.

\section{REFERENCES}

1. F. V. Atkinson, On second order nonlinear oscillation, Pacific J. Math. 5 (1955), 643647.

2. A. Bielecki, Une remarque sur la méthode de Banach-Cacciopoli-Tikhonov dans la théorie des équations differéntielles ordinaires, Bull. Acad. Polon. Sci. 4 (1956), 261-264.

3. A. Constantin, Global existence of solutions for perturbed differential equations, Ann. Mat. Pura Appl. 168 (1995), 237-299.

4. A. Constantin, On the existence of positive solutions of second order differential equations, Ann. Mat. Pura Appl. 184 (2005), 131-138.

5. S. G. Dubé, and A. B. Mingarelli, Note on a non-oscillation theorem or Atkinson, Electron. J. Differential Equations 22 (2004), 1-6.

6. M. Ehrnström, Positive solutions for second-order nonlinear differential equations, Nonlinear Anal. 64 (2006), 1608-1620.

7. M. Ehrnström, Prescribed asymptotic behaviour of solutions to semilinear ordinary differential equations, Appl. Math. Lett., to appear.

8. M. Ehrnström and O. G. Mustafa, On positive solutions of a class of nonlinear elliptic equations, Nonlinear Anal., to appear.

9. T. Hallam, Asymptotic integration of second order differential equation with integrable coefficients, SIAM J. Appl. Math. 19 (1970), 430-439.

10. T. Kusano and M. Naito, Unbounded nonoscillatory solutions of nonlinear ordinary differential equations of arbitrary order, Hiroshima Math. J. 18 (1988), 361-372.

11. T. Kusano, M. Naito and H. Usami, Asymptotic behavior of solutions of a class of second order nonlinear differential equations, Hiroshima Math. J. 16 (1986), 149-159.

12. O. Lipovan, On the asymptotic behaviour of the solutions to a class of second order nonlinear differential equations, Glasgow Math. J. 45 (2003), 179-187. 
13. O. G. Mustafa, Initial value problem with infinitely many linear-like solutions for a second-order differential equation, Appl. Math. Lett. 18 (2005), 931-934.

14. O. G. Mustafa and Y. V. Rogovchenko, Global existence of solutions with prescribed asymptotic behavior for second-order nonlinear differential equations, Nonlinear Anal. 51 (2002), 339-368.

15. O. G. Mustafa and Y. V. Rogovchenko, Asymptotic integration of nonlinear differential equations, Nonlinear Anal. 63 (2005), 2135-2143.

16. Ch. Philos, I. Purnaras, and P. Tsamatos, Asymptotic to polynomials solutions for nonlinear differential equations, Nonlinear Anal. 59 (2004), 1157-1179.

17. H. Usami, Global existence and asymptotic behavior of solutions of second-order nonlinear differential equations, J. Math. Anal. Appl. 122 (1987), 152-171.

18. E. Wahlén, Positive solutions of second-order differential equations, Nonlinear Anal. 58 (2004), 359-366.

19. Z. Yin, Monotone positive solutions of second-order nonlinear differential equations, Nonlinear Anal. 54 (2003), 391-403.

20. E. Zeidler, Nonlinear functional analysis and its applications I: fixed-point theorems (Springer-Verlag, 1985). 